\title{
Mathematical Method for Tracking Ingestible Telemetry Capsule in Real-Time
}

\author{
Francis Adepoju ${ }^{1 *}$ and Khalil Arshak ${ }^{2}$ \\ ${ }^{1}$ School of Information Technology and Computing, American University of \\ Nigeria \\ ${ }^{2}$ Department of Electronic and Computer Engineering, University of Limerick, \\ Ireland \\ ${ }^{1}$ francis.adepoju@aun.edu.ng, ${ }^{2}$ khalil.arshak@ul.ie
}

\begin{abstract}
A detailed analysis of scientific work done in tracking an embedded object with special reference to telemetry capsules in the gastro-intestinal (GI) tract using radio frequency $(R F)$ signals is presented in this paper.

We fully discuss the mathematical analysis of our tracking procedure as well as experimental validation of our tracking concept. Experimental validation is accomplished by using a radio frequency $(R F)$ signals at $433 \mathrm{MHz}$ (Free Industrial Scientific and Medical centre frequency in EMEA regions), Analog Devices 8052 based microcontroller with $A D C$ capability and a means of transferring the received signal strength intensity (RSSI) voltage converted to digital, from the microcontroller to the personal computer $(P C)$. The primary focus of this paper is practical way of achieving these tasks using basic mathematical and computing principles. Consequently, our algorithm-based tracking results for experiments in a liquid medium yielded an average error of less than $21 \%$. These results were validated with the Bland-Altman plots using semi-automated manual measurements as gold standard.
\end{abstract}

Keywords: Gastro-intestinal tract, Trilateration, Wireless Endoscopy, Real -Time Localization

\section{Introduction}

Various technologies have been used to implement wireless positioning systems including ultrasound and radio frequency (RF) identification (RFID). These positioning implementations require specialized hardware and infrastructure and are often expensive. Within the gastro-intestinal (GI) tract for example, traditional methods of radio localization based on time and/or angle based methods (Time Of Arrival, Time Difference Of Arrival and Angle Of Arrival) are not feasible due to the dense multi-path characteristics of the digestive organs. Such a positioning problem is worsened by nonline-of-sight (NLOS) conditions due to intestinal shifting, routing, filling and emptying resulting in intermittent total signal loss. Therefore, to accurately determine the position of an object in the GI tract, simultaneous RSSI measurements from several access points (AP) are needed.

Some previous basic works in the field are reported in [1-5]. Most of these works incorporate devices with radio transmitters, camera, and in some cases, storage. Usually, the device is capable of delivering medication to an identified location in the GI tract. It could identify a specific location based on pictures taken by the camera and, consequently, it could deliver medication or take samples from such identified locations. Other capsules are developed with a facility to measure $\mathrm{pH}$ level temperature and

${ }^{*}$ Corresponding Author 
pressure changes inside the GI tract. With the incorporated camera technology most of the products are able to transmit pictures of the intestine to a base station. However, these devices have no way of determining the physical position of the capsule in real-time, and consequently, their passage through the GI tract are not easily reproducible. Considerable effort around the world has been devoted to exploring the application of micro and nano technology to issues broadly related to human health. There is ongoing research in analyzing more complex biological system such as living cells by combining microfabrication and microfluidic technologies. Various types of embedded cores and miniaturized hardware are gaining significance in clinical medicine for disease diagnosis applications.

The potential applications for an indoor wireless positioning system are many. For example, such systems could be used for an indoor navigation system, or to enable resource management by tracking physical assets and people or to provide a multitude of location based services. Various technologies have been used to implement wireless positioning systems including ultrasound [6, 7], infrared [8] and radio frequency identification (RFID) [9]. These positioning implementations require specialized hardware and infrastructure and are often expensive. Consequently, in medical applications, a wireless positioning implementation with non-specialized, relatively inexpensive hardware is desirable. As demonstrated in this paper, positioning can be implemented by using received signal strength (RSS). Received signal strength is a measure of the power received by a radio receiver from a radio transmitter and provides information about the proximity of the transmitter. Indeed, RSS intensity is location dependent because it is affected by factors such as distance from the transmitter and attenuation due to medium of propagation and other barriers. Useful RSSI ranges are usually between $-105 \mathrm{~dB}$ and $-50 \mathrm{~dB}$ in radio communication [10]. Consequently our experimental results are confined to this range.

The remainder of this paper is organized as follows: Section 2 discusses biological tissue and antennae and the relationship between them at $433 \mathrm{MHz}$; Section 3 introduces our method for performing tracking on the human GI tract model; Section 4 presents the mathematical methods employed to achieve the object tracking; and finally, the results are summarized in the conclusions section.

\section{Biological Tissue \& Antennae}

There are two primary requirements for antennas for on-body links. First the antenna needs to be insensitive to the proximity to the body; second, the antenna needs to have a radiation pattern shape that minimizes the link loss [11]. The matching of antennas is naturally affected by their proximity to the body, due to the influence of the body on the antenna reactive fields. There are various ways of matching antennas. These are discussed in [12]. Antenna matching is outside the scope of this paper but a basic understanding of the subject is necessary to the success of the tracking procedure.

It is to be noted that at ultra high frequencies (UHF) and microwave frequencies, body-worn antennas can suffer from reduced efficiency due to electromagnetic absorption in tissue, radiation pattern fragmentation and variations in feed-point impedance. The significance and nature of these effects are system specific and depend on the operating frequency, propagation environment and physical constraints on the antenna itself. However, biological tissue is, for practical purposes, non-magnetic with permeability $\boldsymbol{\mu}\left(\mathrm{H} \mathrm{m}^{-1}\right)$ close to that of free space [13]. Interaction between radio frequency electromagnetic waves and living systems therefore requires consideration of the influence of both external and internally induced fields. Energy exchanges may occur with either free charges or dipolar (asymmetrically charged) structures such as water. In a rapidly alternating field, free charges may be accelerated, giving rise to conduction currents and corresponding resistive losses, while dipoles suffer reorientation, producing 
frictional resistance caused by the viscous biological medium. Both phenomena result in tissue temperature rise and account for the bulk absorption of RF energy by the biological body. The absorption effects vary in magnitude with both the frequency of the applied field and the characteristics of the tissue material, which is largely based on water and ionic content. Consequently, biological tissues suffer wavelength shortening and raw attenuation as well as reflections at interface boundaries. These factors are usually of utmost importance when studying wave propagation in tissue due to an embedded RF transmitter at a certain frequency $\boldsymbol{f}$. The accumulated effects of the above factors are known to cause a reduction in radiated power from the body [14].

Wavelength shortening and raw attenuation are not the only factors of concern for wave propagation and object detection in tissue due to an embedded RF transmitter (capsule) at a frequency $f$. There usually are reflections at material boundaries, which further reduces radiated power from the body. For a normally incident wave on a dielectric material boundary, the reflection coefficient $\Gamma$ is given by equation (1):

$$
\Gamma=\frac{\eta_{2}-\eta_{1}}{\eta_{2}+\eta_{1}}
$$

where $\eta_{1}$ is the intrinsic complex impedance of the incident medium and $\eta_{2}$ is the intrinsic complex impedance of the medium beyond the boundary [15]. The intrinsic impedance of a dielectric medium can be calculated from the material parameters using equation (2):

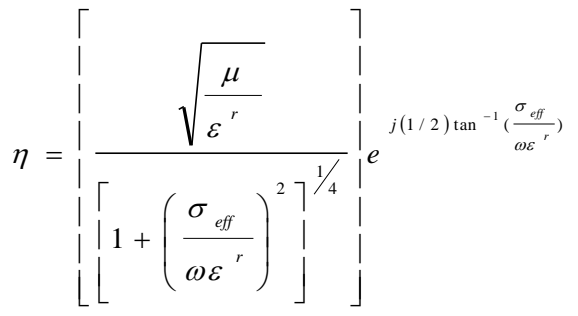

where eff is the effective conductivity, ${ }^{r}$ is the relative permittivity and is

\section{frequency}

Usually for a lossy material like tissue, it is the dissipated power rather than the quality (Q-value), that is the most important quantity in the design of an efficient antenna. Table 1 [6] shows the average transmission factor for normal RF signal incidence at the selected boundaries at $403 \mathrm{MHz}$.

Table 1. Transmission Factor for Normal Wave Incidence (403MHz)

\begin{tabular}{|c|c|c|}
\hline Interface & $\begin{array}{c}\text { Field Reflection } \\
\text { Coefficient }\end{array}$ & $\begin{array}{c}\text { Power Transmission } \\
\text { Factor (\%) }\end{array}$ \\
\hline Muscle to Fat & 0.41 & 83.2 \\
\hline Fat to Skin & 0.37 & 86.3 \\
\hline Skin to Air & 0.78 & 39.2 \\
\hline
\end{tabular}

For a lossy material it has been shown that the optimal value of the radiation efficiency $\left(\boldsymbol{\eta}_{\text {eff }}\right)$ for any antenna is the one obtained for a magnetic dipole [16]. It is notable that the optimal value of the directivity of an electric or a magnetic multi-pole antenna of order one, i.e., a dipole antenna, is 1.5 . This value is the same as the directivity of each partial wave antenna of orders one. For higher order antennas the directivity of a partial wave antenna of order $n$ is always smaller than the maximum directivity of the multi-pole antenna of order $n$ [16]. The radiation efficiency, the directivity and effective power of the transmitting antenna inside the capsule (a dipole) are therefore utilized at the receivers for tracking purposes to ensure optimum link between the transmitter and the 
receivers. Ingestible or implanted devices may be powered for some short-term applications by integral batteries, or they may utilize inductive coupling to charge a capacitor for burst operation. As the operating frequency is increased the efficiency of such compact antenna increases rapidly as they become less electrically small. Above $500 \mathrm{MHz}$, the absorption of electromagnetic energy by the human body increases rapidly due to the high losses caused by the high water content of body tissues [17]; this may result in overall lower telemetry system efficiency at frequencies beyond $500 \mathrm{MHz}$.

At the heart of many positioning methods is the mathematical concept of triangulation: Triangulation is essentially the use of the properties of triangles to calculate distances. Because of its significance in modern positioning methods, a basic description is given here. Originally, triangulation was used for surveying [18] and civil engineering purposes, and later for finding the range of targets for artillery strikes. Given any two reference points it is possible to calculate the distance from one reference point to an object with knowledge of the angles between both references and the object and also the distance between the reference points.

Given any two reference/access points $(\mathrm{AP})$ denoted $\left(\mathrm{AP}_{1}, \mathrm{AP}_{2}\right)$, it is possible to calculate the distance from one reference point to an object with knowledge of the angles between both references and the object and also the distance between the access points. With this amount of information, distances from the object can be computed. These distances represent the radii of circles drawn from the receivers at the specific access points to the location of the object. As shown in Figure 1, three of such circles intersecting will provide the true position of the object in 2-D. If the distances from one access and the neighboring ones are known, it is possible to fully determine the resulting triangle and as such, the real-time location of the object. The solution presented in this paper is based on a modified form of triangulation, called trilateration, whereby there is in no need for angular calculations. This method is found to be suitable for adaptation into telemetry capsules for tracking purposes [6].

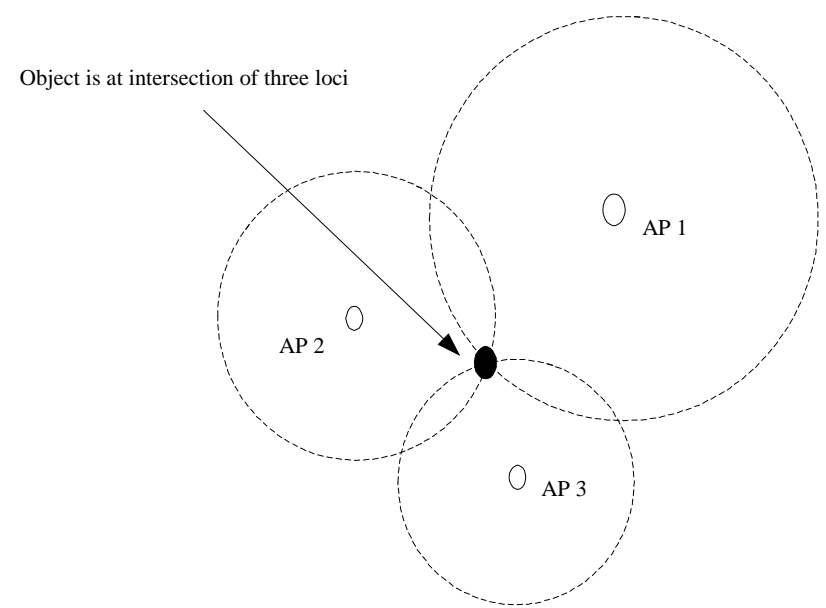

Figure 1. Object Location in 2-D

\section{Solution to the Tracking Problem}

The following section gives full details of how to solve the tracking problem in the GI tract. Schematic diagrams of the arrangement, both experimental and model, are as shown in Figure 2. This illustrates the general overview of the entire concept from data capture through data processing, and to translation of data into graphical form on a personal computer.

As shown in the model view of Figure 2(a), four receivers (Pods) are placed on a predetermined position on the patient's abdomen. In the experimental setup shown in Figure 
2(b), the Pods are wired onto an industry standard Data logger with adequate memory to save location data coming from the microprocessor over the time range of investigation. Consequently at the end of investigation, the saved data are uploaded to a PC running custom software (algorithm). All computation, approximation and compensations are made at this stage. The Analog Devices ADuC841 (or better) was used for the prototype design.

Some of the features of the device that made it suitable for this application are as follows:

- A four channel analog inputs, a high performance self calibrating multi-channel ADC (having a dynamic range of $0.6 \mathrm{mV}$ for an internal VREF of $2.5 \mathrm{~V}$ ),

- A dual DAC, and an optimized single-cycle $20 \mathrm{MHz}$ 8-bit MCU (8051 instruction set compatible), etc.

- These facilitated the collection of weak RF signals and processing them into corresponding voltages (RSSI) using the 12-bit ADC hardware.

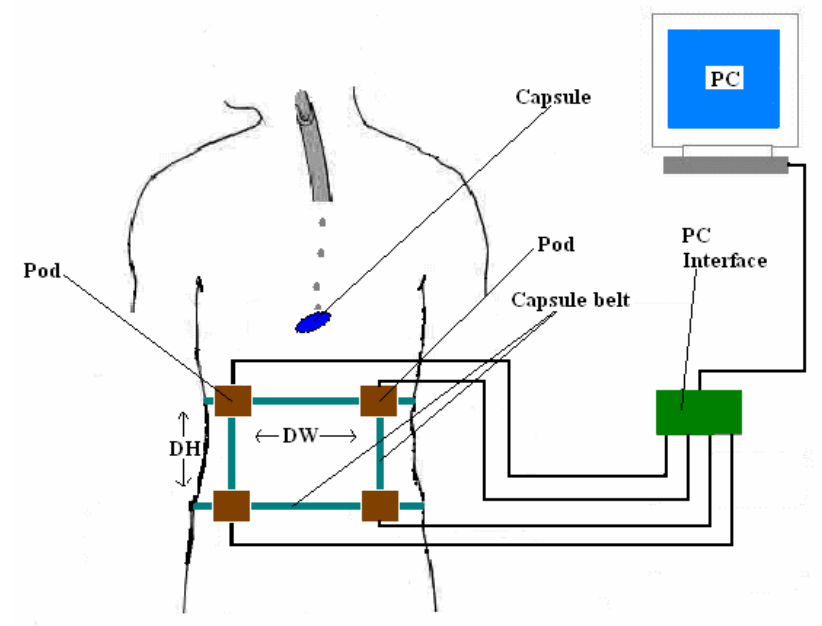

Figure 2(a). Model View - Capsule Tracking with RSSI Output

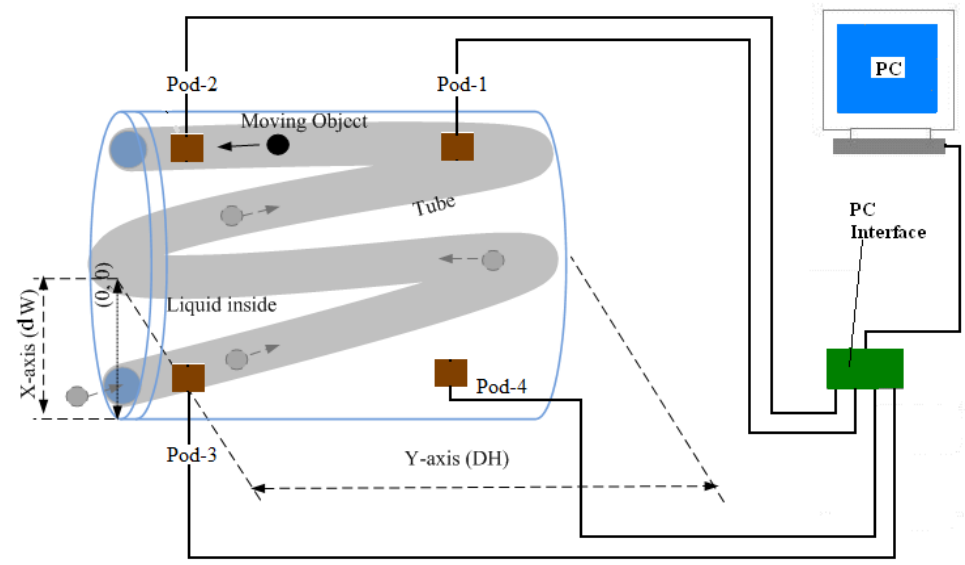

Figure 2(b). Experimental - Capsule Tracking with RSSI Output

At the data collection stages, there are two established methods to obtain the needed positional reference or a-priori position information needed for the algorithm. These are:

1. Through actual measurements - map based propagation model or 
2. Through probabilistic distribution function (PDF) - mathematical deterministic propagation models.

In map based propagation model, approximate distance measurements are made from the fixed Pod positions to some pre-determined, capsule positions. This is done by arbitrarily selecting the coordinates of the capsule inside the cylinder model. Real distances are then calculated based on the approximate distances using the non-linear least square methods. This method consists of a database of RSS indicator vector measurements associated with their corresponding locations. The object can be positioned using the RSSI vector measurement and any of known classification techniques. Radio map propagation model require a burdensome data collection phase where a large number of signal strength measurements must be recorded along with the corresponding location [9]. If the location of the APs or the environment changes then the data collection process must be repeated, because the data due to the old configuration would have become invalid.

It would be preferable to be able to predict RSS values with a propagation model rather than having to manually collect them as explained above. Although the focus of previous research has been mainly on radio map type algorithms, which avoid radio propagation theory and treat the location estimation problem as a classification problem of associating a RSSI vector to a location. Among the work already done using indoor propagation models, little research has been published in applying probabilistic indoor propagation models to positioning algorithms. For example, [19-21] all use deterministic propagation models. In [22], the proposed algorithm estimates the client's position by first determining a maximum-likelihood estimate for the client's distance from each AP using the derived probability distribution function of the received power. These distance estimates along with the known locations of the APs are then used to trilaterate the estimated position of the object. As stated above, the location of capsule can be estimated from the voltage strengths at the receivers. The voltages are translated into distance in the relevant section of the trilateration algorithm.

In our implementation, an initial position location vector is generated based on information from the RSSI voltages and the mathematical method of Linear Least squares. This starting vector (a-priory information) is used to compute the initial position, which is supplied to the Non-Linear least squares algorithm to iteratively compute the most feasible location using the Newton's iteration. An integral part of the work presented in this paper is a fully developed C++ application consisting of four major interrelated programs for achieving capsule triangulation [22]. The approach that we employed is not to solve for object position based on the intersection of 3 circles, but to use the linear approximation techniques on the data received from RSSI conversion process to predict the position of the object and based on the initial prediction, the final position of the object can be computed. As discussed above, we employed the method of trilateration for the object position tracking. Indeed, this approach facilitates the implementation of fully automated real-time position measurements by eliminating the need to measure angles.

The fact that the geometric volume of the target (human torso) is fairly small in space makes the analysis easier since for practical purposes, analysis in 3-D could be implemented by simply eliminating the third axis (z-axis). This consequently reduces the problem to a 2-D analysis problem. Capsule position is determined by considering the magnitude of the received RSSI signals at the four APs respectively located inside the four quadrants of the cylindrical volume representing the abdomen.

As illustrated in Figure 3, both the Pod position and the capsule positions/locations are configured with respect to $\mathrm{N}_{0}$ at $(0,0)$ in software. This facilitates the partitioning of the cylinder model into four quadrants in order to simplify sundry geometric computations. Using the translated distances from the RSSI voltages at the Pods, the algorithm is able to 
determine the approximate value of $\mathrm{r}_{\mathrm{ni}}$. This is later utilized by the Non-Linear Least Squares methods to compute the precise location of the capsule.

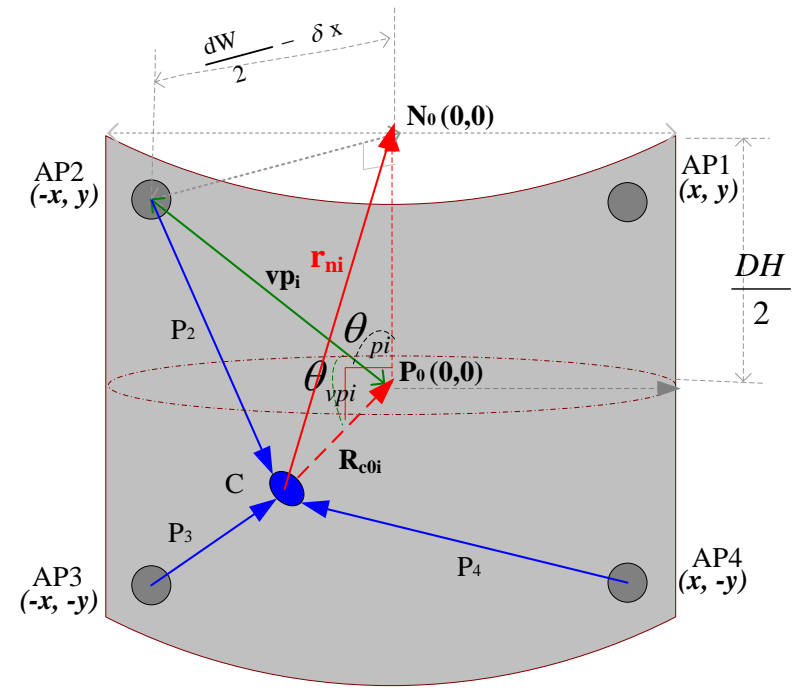

Figure 3. Determination of Capsule Position Relative to Origin, $N_{0}(0,0)$

\section{Mathematical Analysis}

Given $n^{\text {th }}$ sequence of capsule location at any time as $n$, and the $i^{\text {th }}$ Pod as $i$ then, while $\mathrm{n} \geq 0$ and for $\mathrm{i}=1 \ldots .4$;

1. The fixed position Pods for collecting data are labeled $\mathrm{AP}_{i}$. The threedimensional coordinates of the capsule, $(x, y, z)$, stand for the approximated coordinate of the capsule in any of the quadrants and the real coordinate of the pods are represented by $\left(x_{i}, y_{i}, z_{i}\right)$. Such coordinate positions will be represented as $\mathrm{P}_{i}$.

2. The approximate distance between any access point and the capsule is represented by the vectors $r_{i}$. For the exact distances, these are simply shown as $\vec{r}_{i}$

3. The 2-Dimensional coordinates of the time-related positions of the capsule in the GI tract, which are unknown in the abdominal space are denoted as $x, y$ and are represented by $P_{n}(x, y)$.

4. The knowledge of $P_{n-1}$ and that of $P_{n}$ are combined to tract the trajectory of the capsule within the period of non-invasive examination.

If the 3 coordinates $x, y, z$ are considered, the exact distance $\vec{r}_{i}$ (radii) is calculated from the approximate values and the coordinates of the Pods $\left(x_{i}, y_{i}, z_{i}\right)$ as equation (3):

$$
\vec{r}_{i}=\sqrt{\left(x-x_{i}\right)^{2}+\left(y-y_{i}\right)^{2}+\left(z-z_{i}\right)^{2}} \quad i=1,2,3,4
$$

The coordinates of the capsule is denoted as $x, y, z$. (from any arbitrary reference origin $(0,0,0))$

Determining these unknown coordinates is our first main task: 


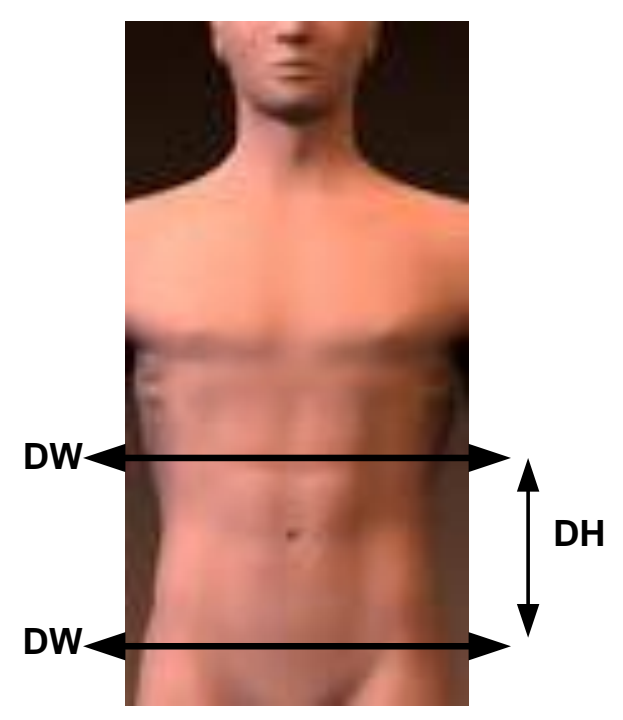

Figure 4. Dimension of Abdomen for Cylinder Model

As shown in Figures 2 and 3, each Pod has pre-determined position vectors $r_{i}$ dependent on the physical size of the patient, for some arbitrary capsule location within the GI tract. This is computed in software based on input parameters DW and DH shown in Figure 4. These physical data, together with other modeling parameter data are used to compute the position matrix as shown in the next section.

The general way of solving this kind of positioning problem is to treat the coordinates of the capsule $P(x, y, z)$ as the point of intersection of several spheres, whose centers are the locations of the Pods. The known exact distances between the capsule and the Pods, $r_{i}$, are the radii of the individual spheres. The equation for any of these spheres is given by equation 3 as:

$$
\vec{r}_{i}=\sqrt{\left(x-x_{i}\right)^{2}+\left(y-y_{i}\right)^{2}+\left(z-z_{i}\right)^{2}} \text { or } \vec{r}_{i}^{2}=\left(x-x_{i}\right)^{2}+\left(y-y_{i}\right)^{2}+\left(z-z_{i}\right)^{2}
$$

The point of intersection of the surfaces of $n$ of these spheres is obtained by letting $i=$ $1,2,3 \ldots n$, and solving the resulting $n$ non-linear equations simultaneously to eliminate two coordinates. This solution technique is quite involved because it produces a nonlinear equation of high degree $(5$ for $n=4)$. Furthermore, since the equations are quadratic, many cases for sign changes would have to be considered.

The approach presented in this paper is to linearize the system of equations. Mathematically, this will reduce the degree of equation, and convert the problem into one of finding the point of intersection of several planes. When the exact distances $\vec{r}_{i}$ from the Pods are available, the solution of the resulting system of equations is fully determined. In our case, there will be 2 equations and 2 unknowns when any combination of 3 Pods is used to produce a 2-D position solution or 3 equations with 3 unknowns when any combination of 3 Pods are used to produce a 3-D position solution - see future works.

We shall now briefly discuss the basic conversion processes for transforming the tracking data through various stages of the algorithm that are fully discussed in [12].

\section{A. RSSI-to-distance Conversion is Implemented as Follows:}

The starting point is to obtain the initial approximate distance by using data obtained from the RSSI signals. 
As stated above, the location of capsule is estimated from the voltages at the receivers. The voltages are translated into distance in the relevant section of the custom algorithm by using equations $(5,6)$ :

$$
\operatorname{RSSI}(\mathrm{V})=v_{\text {bat }} \cdot \frac{A D C-\text { Counts }}{2^{n}} .
$$

where $V_{\text {batt }}=2.5 \mathrm{~V}$ (Internal Voltage Reference)

ADC_Counts $=$ Processor counts of data events using transceivers connected to a suitable $\mathrm{ADC}$ and $n=$ resolution of the ADC.

If source impedance is $50 \Omega$ and $n=12$ as is the case in this work, then equation (5), through the inverse square law, can be reduced to distance as:

$$
r=d=\sqrt{\frac{R}{V^{2}}}=\frac{7.07}{V_{(R S S I)}}
$$

As shown in Figure 5, it is possible to use the cosine rule to compute the length of intestinal segment which the capsule have traversed between the time periods $\mathbf{t}_{\mathbf{i}-1}$ and $\mathbf{t}_{\mathbf{i}}$. The end result of all data collection, transformation and manipulation is a final human readable version of the data, which can be displayed graphically on the computer screen for the physician to interpret.

\section{B. The Least Squares Method}

The 3-D location of the capsule is first approximated by transforming the magnitude of the strength of pulse signals received at the Pods to distance. For 2-D localization, only 3 Pods are selected to determine the approximate coordinate. These are usually the combination of any of 3 Pods with the largest voltage magnitude, indicative of the fact that the capsule is somewhere around the vicinity of those Pods.

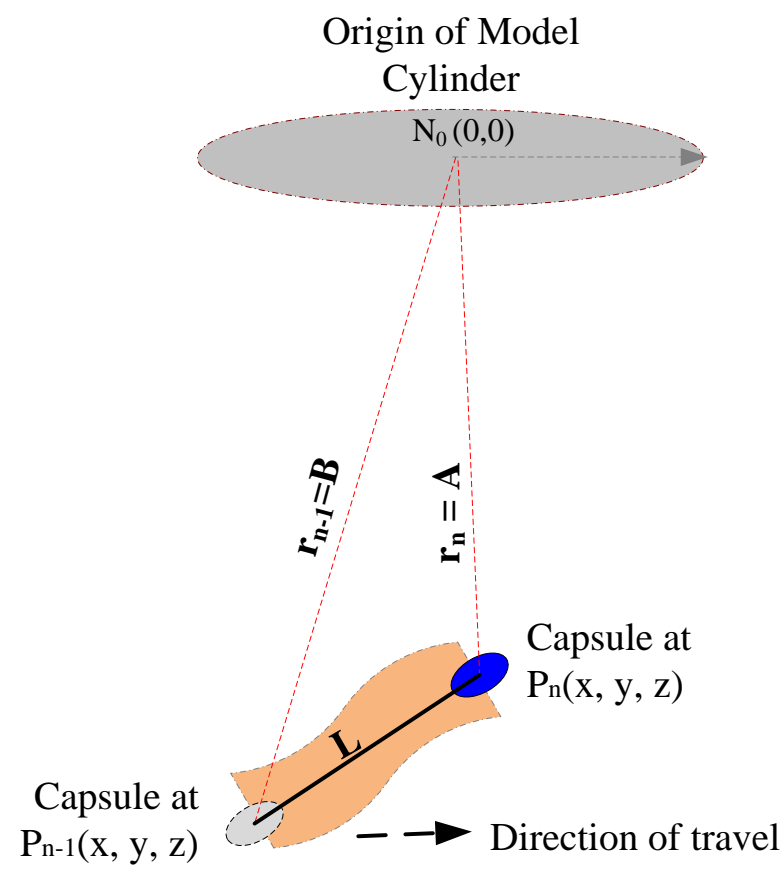

Figure 5. Successive, Accumulated Intestinal Length Computation

The algorithm is programmed for the above operations. Of course, the data are sorted in order of magnitude before usage in order to unambiguously map the four Pods to ADC 
channels 1 to 4 . The resulting linear system can easily be written in matrix form as equation (7):

$$
A \vec{x}=\vec{b}
$$

with

$$
\begin{gathered}
A=\left(\begin{array}{lll}
x_{2}-x_{1} & y_{2}-y_{1} & z_{2}-z_{1} \\
x_{3}-x_{1} & y_{3}-y_{1} & z_{3}-z_{1} \\
x_{4}-x_{1} & y_{4}-y_{1} & z_{4}-z_{1}
\end{array}\right) \\
\vec{x}=\left(\begin{array}{l}
x-x_{1} \\
y-y_{1}
\end{array}\right) \\
\left(\begin{array}{l}
z-z_{1}
\end{array}\right) \\
\vec{b}=\left(\begin{array}{l}
b_{21} \\
b_{31} \\
b_{41}
\end{array}\right)
\end{gathered}
$$

where $\vec{b}$ is directly related to $r_{j}, r_{i}$ and the distance between Pods $i$ and $j$. The linear system of equation (7) has 3 equations in 3 unknowns. Therefore, theoretically only four Pods are needed to determine the unique position of the capsule in 3-D or, as presented in this work, three Pods are needed in order to determine the unique position of the capsule in 2-D.

\section{Solution Constraints:}

Given a square complex or real matrix $\mathrm{A}$, a matrix norm $\|\mathrm{A}\|$ is a nonnegative number associated with A fulfilling the condition that $\|\mathrm{A}\|>0$ when $\mathrm{A} \neq 0$ and $\|\mathrm{A}\| 0$ iff $\mathrm{A}=0$. The most frequently used norms in linear algebra are the Frobenius norm [23]; given by:

$$
\|A\|_{F}=\sqrt{\sum_{i=1}^{m} \sum_{j=1}^{n}\left|a_{i j}\right|^{2}}
$$

i.e. Square root of the sum of squares of the matrix elements

A well-conditioned matrix has a finite determinant; whereas an ill-conditioned matrix has a non valid determinant; i.e., singular matrix with a zero in the denominator. The transpose of matrix $\mathrm{A}$ is denoted by $\mathrm{A}^{\mathrm{T}}$

For example, Let $\vec{A}$ be any $2 \times 2$ matrix formed from A. The validity of the solution will partly depend on the condition number $c(\vec{A})$, which is defined as the product of the norm of $\vec{A}$ and the norm of the inverse of the coefficient matrix $\vec{A}$, given by equation (12):

$$
c(\vec{A})=\|\vec{A}\|\left\|\vec{A}^{-1}\right\|
$$

The equations should be "well conditioned" in order to be able to determine all components of $\vec{x}$ with a good accuracy. The change $\delta \vec{x}$ of the solution $\vec{x}$ resulting from the changes of $\vec{A}$ and $\vec{b}$ [24] is given by equation (13):

$$
\frac{\|\vec{\delta}\|}{\|\vec{x}\|} \leq M c(\vec{A})\left(\frac{\|\overrightarrow{b \vec{b}}\|}{\|\vec{b}\|}+\frac{\|\delta \vec{A}\|}{\|\vec{A}\|}\right)
$$


where $M=\frac{1}{1-\alpha}$, with $\alpha=\left\|(\delta \vec{A}) \cdot \vec{A}^{-1}\right\|<1$. Both the data $\vec{b}$ and the position of the APs (reflected in $\vec{A}$ ) can be modified to optimize the calculation of the position $\vec{x}$ of the capsule.

Mathematically, the coordinates of positions obtained by applying the linear least squares method to the linear system of equation (7) are generally more accurate than the coordinates obtained by solving four equations from the linearized system of equations ( 3 or 4) directly [24].

However, since the distances $\mathrm{r}_{\mathrm{i}}$ are only approximates, the problem requires the determination of $x$ such that $A \vec{x} \approx \vec{b}$.

\section{Minimizing the Residuals:}

Minimizing the squares of the residuals $\vec{b}$.- $A \vec{x}$.yields equation (14):

$$
S=\vec{r}^{T} \vec{r}=(\vec{b}-A \vec{x})^{T}(\vec{b}-A \vec{x}) \quad,
$$

which leads [26] to the normal equation: $A^{T} A \vec{x}=A^{T} \vec{b}$

There are several methods to solve equation (15) for $x$.

According to [24] the condition number of $\mathbf{A}^{\mathrm{T}} \mathbf{A}$ determines which method is best.

- If $\mathbf{A}^{\mathbf{T}} \mathbf{A}$ is non-singular and well conditioned, then the solution [26] of $A^{T} A \vec{x}=A^{T} \vec{b}$ is given by equation (16):

$$
\vec{x}=\left(A^{T} A\right)^{-1} A^{T} \vec{b}
$$

- However, if $\mathbf{A}^{\mathrm{T}} \mathbf{A}$ is singular ( $\left.\operatorname{det}(\mathrm{A})=0\right)$ then the normalized $\mathrm{QR}$ decomposition [25] of A could be used to solve for $\vec{x}$ [QR decomposition is not referenced beyond this point in this work].

In reality, a "Bad record" exception is thrown by the custom algorithm whenever $\mathbf{A}^{\mathrm{T}} \mathbf{A}$ resolves to zero. In that case, processing will proceed to the next record. In some cases, the location estimate $\mathrm{x}$ may not correspond to a feasible physical position, i.e. the estimate may lie outside the cylinder model space of Figure 4. In those cases, the location estimate is usually replaced by the closest (in the cylindrical model space) feasible location. This scenario is fully documented in the algorithm presented in [22].

Various methods were discussed in [27] to solve the system of linear equation (7). However, due to heavy dependence on approximate distances $\left(r_{i}\right)$ in the tracking problem, we decided to adhere to the method of non-linear least squares to solve the system of linear equations. The main constraint on this solution is that the capsule must not escape outside of the cylindrical space. Also, due to the relatively small size of the resulting cylinder model, it is possible to work in 2-D, by approximating the z-axis to zero. So the four Pods determine the 2-D coordinate of the capsule based on a selection of three out of four Pod data where RSSI signals are greatest. The logic of this approach is that the receivers closest to the physical location of the capsule will most certainly detect a signal with stronger power level than those well away from the capsule.

Non-linear least squares method is based on the fact that the sums of the squares of the errors on the distances are minimized. This solution yielded a reasonably accurate result even when the distance error in $r_{i}$ is appreciable.

Recall that $r_{i}$ denotes the approximate distance between the capsule and the $i^{\text {th }} \mathrm{Pod}$; and that $\vec{r}_{i}$ stands for the exact, unknown distance, i.e. from equation 4 , we arrived at: $\vec{r}_{i}^{2}=\left(x-x_{i}\right)^{2}+\left(y-y_{i}\right)^{2}+\left(z-z_{i}\right)^{2}$ 
Therefore, in order to minimize the sum of squares of the errors on distances, we need to minimize the function given by equation (17):

$$
F(x, y, z)=\sum_{i=1}^{n}\left(\bar{r}_{i}-r_{i}\right)^{2}=\sum_{i=1}^{n} f_{i}(x, y, z)^{2}
$$

with

$$
f_{i}(x, y, z)=\vec{r}_{i}-r_{i}=\sqrt{\left(x-x_{i}\right)^{2}+\left(y-y_{i}\right)^{2}+\left(z-z_{i}\right)^{2}}-r_{i}
$$

where $f_{i}$ is the square of error in coordinate distance at point $i$. Minimizing the sum of the square errors is a fairly common problem in applied mathematics for which various algorithms are available [28]. Newton's iteration was selected from among the numerous methods to find the 'optimal solution' $P(\vec{x}, \vec{y}, \vec{z})$ [29]. To do this, a good initial guess for $(x, y, z)$ is obtained from the linear square method of equation (7). The only case considered in this work is the case for which the lowest value of $\mathrm{F}(\mathrm{x}, \mathrm{y}, \mathrm{z})$ - the derivative of $f(x, y, z)$ given by $f_{i}\left(\mathrm{~F}_{\mathrm{min}}\right)>0$, i.e., positive.

Differentiating equation (17) with respect to $x$ yields

$$
\frac{\partial F}{\partial x}=2 \sum_{i=1}^{n} f_{i} \frac{\partial f_{i}}{\partial x}
$$

The formulae for the partials with respect to $\mathrm{y}$ and $\mathrm{z}$ are:

$$
\begin{aligned}
& \frac{\partial F}{\partial y}=2 \sum_{i=1}^{n} f_{i} \frac{\partial f_{i}}{\partial y} \\
& \text { and } \frac{\partial F}{\partial z}=2 \sum_{i=1}^{n} f_{i} \frac{\partial f_{i}}{\partial z} \text { respectively. }
\end{aligned}
$$

The solution to the linear system $A \vec{x}=\vec{b}$ can be obtained starting with $\mathrm{P}_{0}$, and using a suitable iteration scheme, i.e., Newton's iteration. If $\mathrm{P}_{0}$ is carefully chosen, a sequence $P_{\{k\}}$ is generated which converges the solution P, i.e. AP $\square$ b . A sufficient condition for the method to be applicable is that A is strictly diagonally dominant or diagonally dominant and irreducible.

The matrix A is strictly diagonally dominant if:

$$
\left|a_{i, i}\right| .>\sum_{j=1}^{i-1}\left|a_{i, j}\right|+\sum_{j=i+1}^{n}\left|a_{i, j}\right| \text { for } i=1,2, \ldots n
$$

\section{$a_{i, i}=$ diagonal elements, and $a_{i, j}=$ off diagonal matrix elements}

Solution via Jacobi iteration depends on the number of iterations and the required accuracy. As implemented in the custom miapsNLLS.cpp program [22], the algorithm is constrained around a maximum of 10 iterations and a configurable accuracy, i.e., $20 \%$.

Introducing vectors $\vec{f}, \vec{g}$ and the Jacobian matrix $\boldsymbol{J}$, equations (17 - 21) translates to:

$$
\vec{g}=2 J^{T} \vec{f}
$$

where 


$$
\begin{aligned}
& J=\left|\begin{array}{lll}
\frac{\partial f_{1}}{\partial x} & \frac{\partial f_{1}}{\partial y} & \frac{\partial f_{1}}{\partial z}
\end{array}\right|, \\
& \bar{f}=\left(\begin{array}{l}
f_{1} \\
f_{2} \\
f_{3}
\end{array}\right), \\
& \vec{g}=\left|\begin{array}{l}
\frac{\partial F}{\partial x} \mid \\
\frac{\partial F}{\partial y} \mid \\
\frac{\partial F}{\partial z}
\end{array}\right|
\end{aligned}
$$

Introducing the solution vector $\vec{R}=\left|\begin{array}{l}x \\ y\end{array}\right|$

Newton iteration $[24,25]$ gives:

$$
\vec{R}_{\{k+1\}}=\vec{R}_{\{k\}}-\left(J_{\{k\}}^{T} J_{\{k\}}\right)^{-1} J_{\{k\}}^{T} \vec{f}_{\{k\}}
$$

where $\vec{R}_{\{k\}}$ denotes the $k^{\text {th }}$ approximate solution. The subscript [30] in $\boldsymbol{J}$ and $\vec{f}$ means that these quantities are evaluated at $\vec{R}_{\{k\}}$. Obviously $\vec{R}_{\{1\}}=(\vec{x}, \vec{y}, \vec{z})^{T}$, the initial solution obtained with the Linear Square methods.

Denoting the components $\vec{x}$ by $(x, y, z)$. Using the explicit form of function $f_{i}(x, y, z)$, then the matrix $J(\vec{x})^{T} J(\vec{x})$ can be written as equations $(29,30)$ :

$$
J^{T} J=\left\{\begin{array}{ccc}
\sum_{i=1}^{n} \frac{\left(x-x_{i}\right)^{2}}{\left(f_{i}+r_{i}\right)^{2}} & \sum_{i=1}^{n} \frac{\left(x-x_{i}\right)\left(y-y_{i}\right)}{\left(f_{i}+r_{i}\right)^{2}} & \sum_{i=1}^{n} \frac{\left(x-x_{i}\right)\left(z-z_{i}\right)}{\left(f_{i}+r_{i}\right)^{2}}
\end{array} \mid\right.
$$




$$
\text { and } J^{T} \vec{f}=\left\{\begin{array}{c}
\sum_{i=1}^{n} \frac{\left(x-x_{i}\right) f_{i}}{\left(f_{i}+r_{i}\right)} \mid \\
\sum_{i=1}^{n} \frac{\left(y-y_{i}\right) f_{i}}{\left(f_{i}+r_{i}\right)} \\
\sum_{i=1}^{n} \frac{\left(z-z_{i}\right) f_{i}}{\left(f_{i}+r_{i}\right)}
\end{array} \mid\right.
$$

Usually, this type of iteration works fast, in particular when matrix $\boldsymbol{J}^{T} \boldsymbol{J}$ is augmented by a diagonal matrix. This effectively biases the search direction towards that of steepest descent (gradient descent). Consequently as the solution is approached, the modifications can be expected to have a decreasing effect. Equations $(29-30)$ are then used in the Newton's iteration - equation (28).

\section{Error Analysis: The Bland-Altman Plots}

The Bland-Altman plot [31] is a statistical method to compare two measurements techniques $\left(\mathrm{X}_{1}\right.$ and $\left.\mathrm{X}_{2}\right)$. In this graphical method the differences (or alternatively the ratios) between the two techniques are plotted against the averages of the two techniques; i.e. $\left(X_{1}-X_{2}\right)$ vs. $\left(X_{1}+X_{2}\right) / 2$. None of the classical ways of error measurements (regression, correlation, etc.) tells us whether the results of manual measurements and that obtained from the tracking algorithm can be considered equivalent. A generally accepted way of interpreting the plot in order to confirm agreement is to analyze the behavior of the cluster of points around the zero line, and also to examine the percentage of points outside the confidence interval (1.96SD) lines.

\section{Experimental Results}

As shown in Figures 6(a-d), Intestinal length can be computed piece-wise by linearly following the curve of the trajectory with respect to time. Processing of data from the tracking operation was performed on a local host computer (PC). Host computer processing is particularly useful since the algorithm is based on solving a fair amount of matrices at different levels. Such number intensive processing generally suits a dedicated local host computer. Finally, a human readable version of data is generated and displayed on the computer screen for the physician to visually locate a particular section of the intestine based on real-time computed length of intestines. The Bland-Altman plot was used to validate the results obtained since there is no other established reference with which such tracking results could be benchmarked.

Figures 6(a-c) shows the trajectory of object in liquid medium due to the algorithm for three instances of tracking experiments. Figure 6(d) is the graph of object tracking (average of three experimental and manual measurements of capsule position). Likewise, Figures 7 (a-d) show the corresponding Bland-Altman plots as discussed, due to manual and the algorithm (experimental) capsule location measurements. 


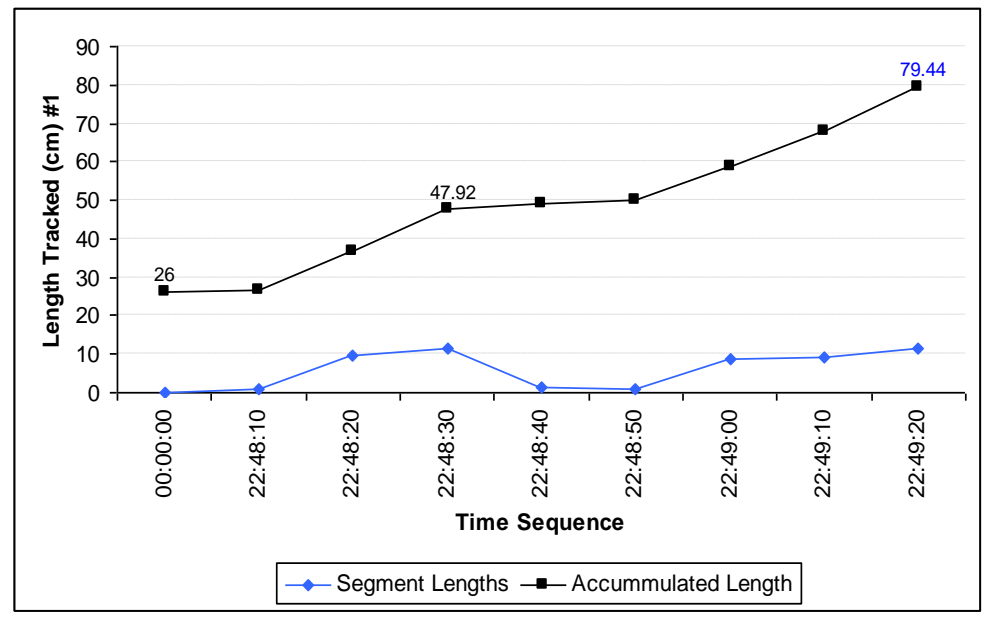

Figure 6. (a) Trajectory of Object in Liquid - Algorithm (Experiment \#1)

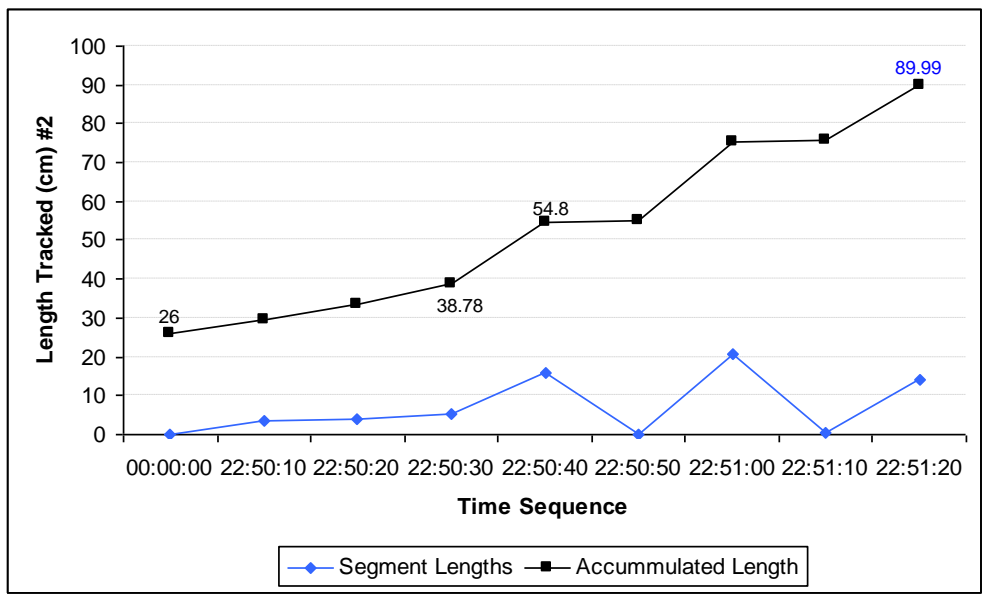

Figure 6. (b) Trajectory of Object in Liquid - Algorithm (Experiment \#2)

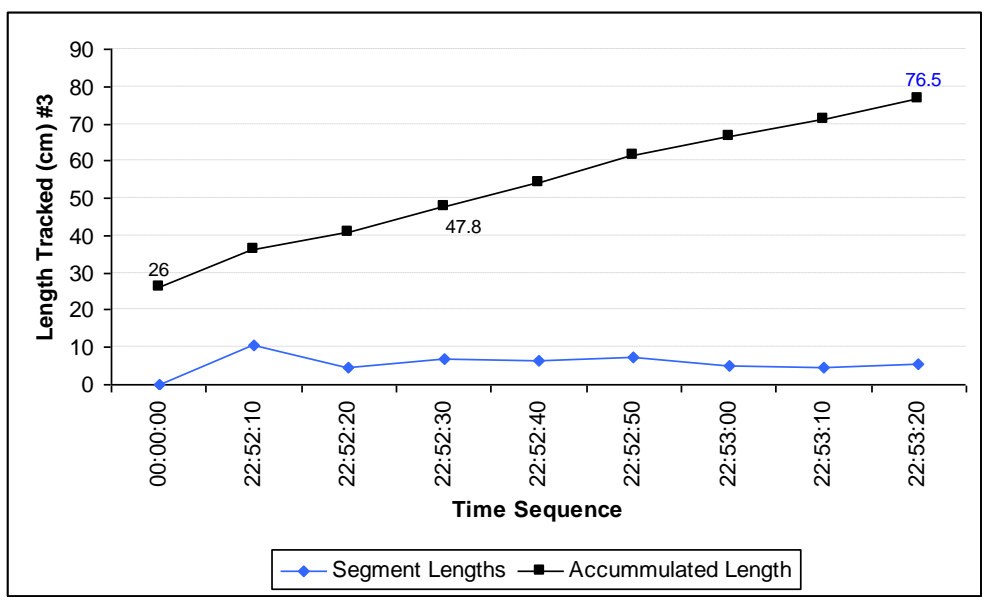

Figure 6. (c) Trajectory of Object in Liquid - Algorithm (Experiment \#3) 


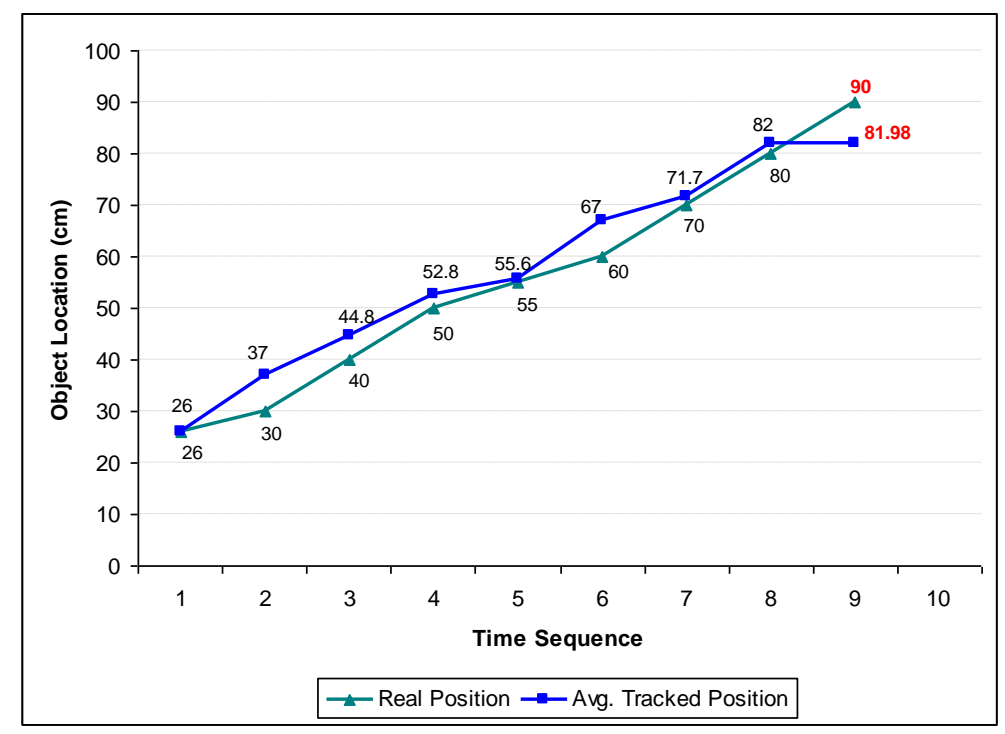

Figure 6. (d) Real Trajectory of Object vs. Average Trajectory of Object

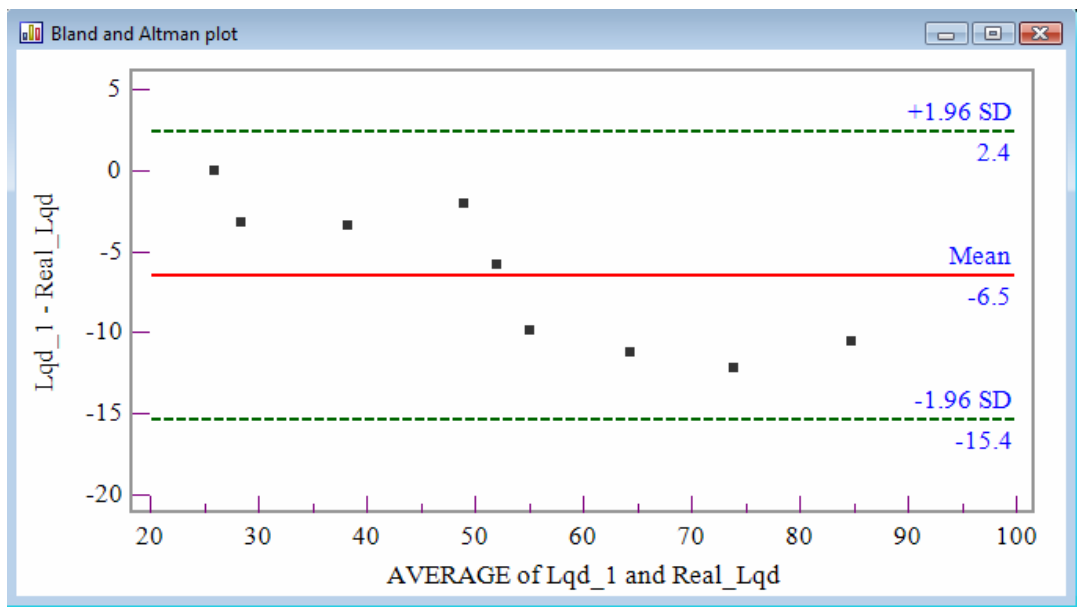

Figure 7. (a) The Bland-Altman Plots for the First Tracking Experiment

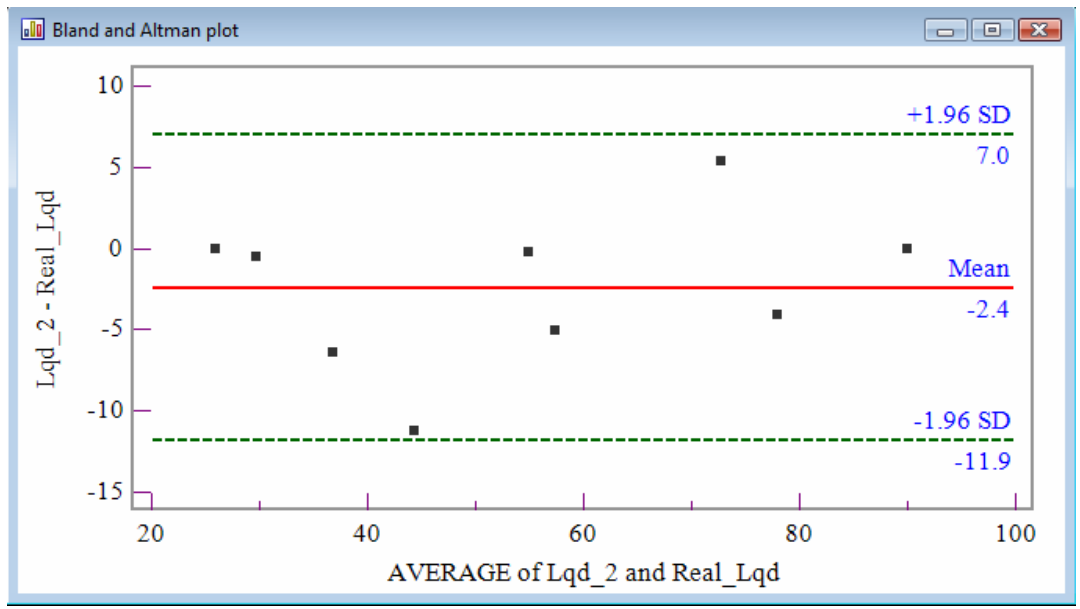

Figure 7. (b) The Bland-Altman Plots for the Second Tracking Experiment 


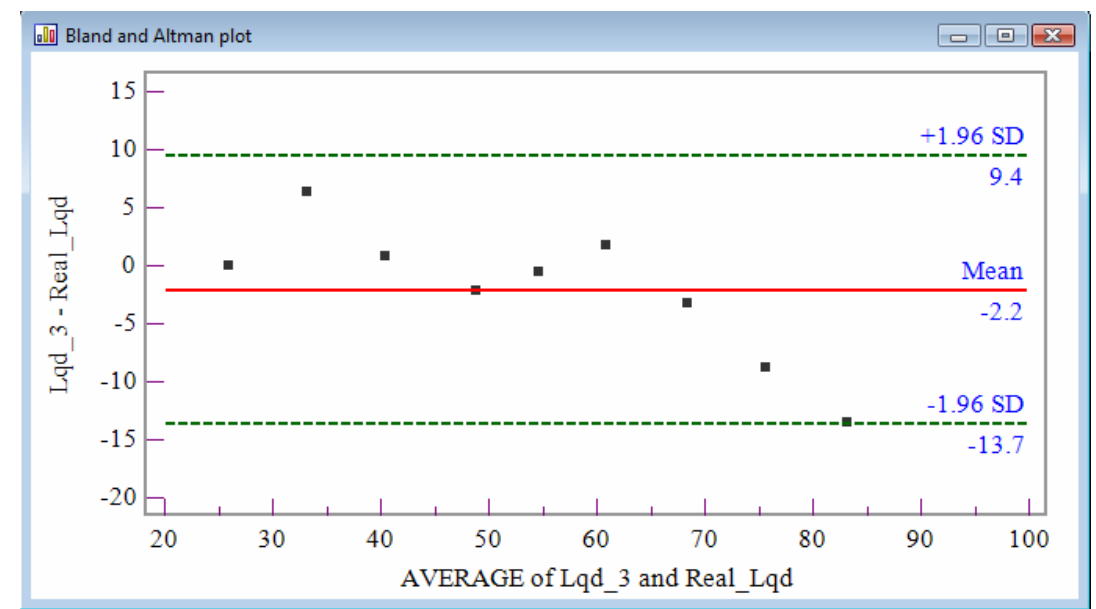

Figure 7. (c) The Bland-Altman Plots for the Third Tracking Experiment

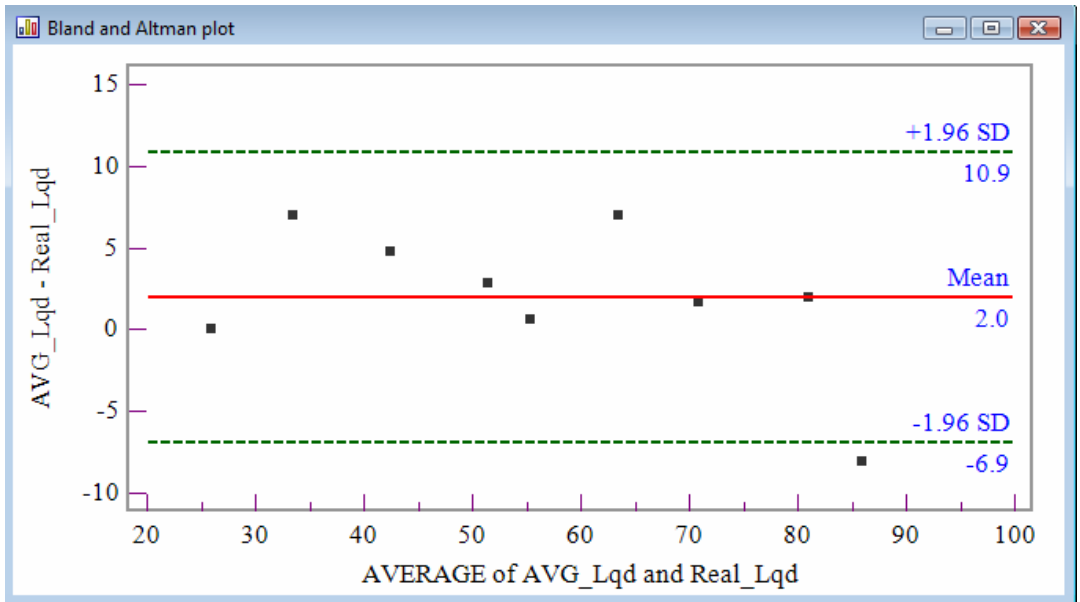

Figure 7. (d) The Bland-Altman Plots for the average of Three and the Real; i.e., the Two Methods of Tracking the Object

\section{Discussion}

Within the last ten years, the Federal Communications Commission (FCC) rules have been modified to meet medical short-distance communication needs. Of course a major limitation of power source continues to plague engineering design of medical short-range devices. However, solutions, such as a design for low duty cycle, the use of repeaters, or body area networks design with efficient power are practical. Wireless telemetry is tipped to make bio-medical measurements more efficient and more reliable and in some cases bring improved health care at a major reduction in cost. This paper described the theory and implementation of a method of tracking an object in inaccessible tubing both in air and also in an environment similar in electrical characteristics to the human GI tract. A custom radio transmitter unit at $433 \mathrm{MHz}$ was used as a test case capsule while the PC interface unit was constructed with adequate electronics for signal capture, signal digitizing and communication with a personal computer.

It was noted that the error in liquid tracking experiments could not be represented intelligently as a ratio. Therefore another way of quantifying the error in tracking had to be used. As discussed in Section 5, none of the classical ways of error measurement (regression, correlation, etc.) could tell us whether the results of manual measurements and that obtained from the tracking algorithm can be considered equivalent. In that case, the Bland-Altman plots could be employed for comparative analysis as explained in the 
previous sections. Consequently the custom algorithm was put to use for tracking the transmitter unit (capsule model). This was accomplished by following the transmitter electronics along the trajectory of the $90 \mathrm{~cm}$ tubing in three different experiments. The segment length and total final tube lengths produced by the algorithm compares quite reasonably with the record obtained by physical measurements with less than $21 \%$ error. From the method comparison graphs shown in Figures 7 (a-c), the systematic biases in the three experiments are obvious. The presence of systematic bias or CB indicates that the test method measures consistently higher or lower in comparison with the reference. This is very true and can be confirmed from Figures 6 (a-c). A systematic adjustment may correct for this by eliminating errors due to measurements and experimental conditions. Using aggregate values as shown in the AVE (Average) plot of Figure 6(d) produced a more acceptable graph with a fairly reasonable negative mean value at the computed standard deviation. There are no outliers, and all test points are decisively captured within the confidence interval.

\section{Conclusion}

An in-depth discussion of a rigorous mathematical method for achieving object tracking which could be extended into tracking ingestible capsules for diagnosing intestinal problems in humans was presented in this paper. A practical implementation of object tracking with RF at very small distance (less than $100 \mathrm{~cm}$ ) was covered. Effects of conditions for variability in RF transmission and reception were discussed and finally, object detection and tracking was demonstrated and the output results from the tracking experiments was shown to be very satisfactory for the target application. The BlandAltman plots were used for comparative analysis on the results from physical measurements and position information, which were produced by the algorithm for the tracking events. The analysis confirmed that the method presented in this paper could be used with fair degree of accuracy to track a capsule using RSSI signals. The inherent imprecision in experimental procedures are equally captured in the Bland-Altman graphs and these do not have any significant effects on the analysis. Also, the Bland-Altman analysis does not rely on number of runs or sample size. However, the results became more meaningful when more than one set of experiments were considered, in which case average of values were used for the comparative analysis.

From the error analysis graphs shown in Section 6, a constant bias was observed in most of the results. In real life experiments, constant biases cannot be totally eliminated but could be minimized. This can be achieved by minimizing the effects of human, environment, instruments, etc. on the measuring process. These sources of error in form of path-loss variables were compensated for inside the accompanying custom algorithm. Subsequently, a maximum error less than $21 \%$ was achieved with three runs of experiments using fluid with similar electrical characteristics as the fluids of the human abdomen as the main medium of propagation. Although the range of position results obtained conforms to theoretical expectations, it is understandable that practical implementation inside the human GI tract will introduce some additional variations in the final results due to the effects of noise, organ-interface reflection, hardware and true nature of the human tissue. Future works will involve the Finite-difference time-domain (FDTD) simulation of the antenna in layers of tissue representing the human organs, validating the radiation pattern with more path-loss variables and validating the tracking algorithm under near-live scenario. A more accurate investigation will also be considered by modifying the algorithms to operate in 3-D.

\section{Acknowledgment}

We express our profound appreciation to the Enterprise Ireland for supporting this project. We are also grateful for the special support and encouragement from the 
department of Electronics and Computer Engineering of the University of Limerick and also to the Mid-Western Health Board, Ireland.

\section{References}

[1] "Method for Delivering a Device to a Target Location", United States Patent - US 2006/0004285A1, no. 6950690, (2006) January 5.

[2] "A tracking system-Real-Time Tracking of A Capsule in the Human GI Tract", United States/International Patent Application Publication \#: 2005/0556, (2005) August 22.

[3] "Multi-Functional, Bi-Directional Communication Telemetry Capsule", United States Patent Application Publication \#:US 2004/0106849 A1, (2004) June 3.

[4] M. B. Ratcliffe, K. B. Gupta, et al., "Use of sonomicrometry and multidimensional scaling to determine the three-dimensional coordinates of multiple cardiac locations: Feasibility and initial implementation", IEEE Trans. Biomed. Eng., vol. 42, no. 6, (1995), pp. 587-598.

[5] F. Adepoju, K. Arshak and D. Waldron, "A review and adaptation of methods of object tracking to telemetry capsules", IC-MED, Int. Journal of Intelligent Computing in Medical Sciences and Image Processing, vol. 1, no. 1, Is. 1, (2007), pp. 35-46.

[6] F. Adepoju and K. Arshak, "Tracking system" US Patent WO 2007/023477A3 or US 20090124871 A1, (2007) March 1.

[7] N. B. Priyantha, A. Chakraborty and H. Balakrishnan, "The cricket location-support system", in 6th ACM International Conference on Mobile Computing and Networking, IEEE Communications Society / WCNC 20052353 0-7803-8966-2/05, (2000) August.

[8] R. Want, A. Hopper, V. F. Ao and J. Gibbons, "The active badge location system," in ACM Transactions on Information Systems, vol. 10, no. 1, (1992) January, pp. 91-102.

[9] L. M. Ni, Y. Liu, Y. C. Lau and A. P. Patil, "Landmarc: Indoor location sensing using active rfid", in First IEEE International Conference on Pervasive Computing and Communications, (2003) March, pp. 407.

[10] M. Robinson and I. Psaromiligkos, IEEE Communication Society, ISBN 0780389662/05.

[11] P. S. Hall and Y. Hao, "Antennas and Propagation for Body-Centric Wireless Communications", Artech House, ISBN-10: 1580534937, (2006).

[12] P. S. Hall and Yang Hao, "Microstrip Patch Antenna with Integrated Adaptive Tunuing", 10th IEE Antennas and propagation Conf, Edinburgh, (1997) April, pp. 506-508.

[13] W. G. Scanlon and N. E. Evans, "Numerical analysis of bodyworn UHF antenna systems", Electronics \& Communication Engineering Journal, vol. 13, Issue 2, (2001) April, pp. 53-64.

[14] R. S. Mackay, "Bio-Medical Telemetry", New York, NY: Wiley, (1970).

[15] W. G. Scanlon, "Analysis of tissue-coupled antennas for UHF intra-body communications", Antennas and Prop, (ICAP), 12th Int Conf (Conf. Publ.), vol. 2, no. 491, (2003) March 31-April 3, pp. 747 - 750.

[16] A, Karlsson, "Physical Limitations of Antennas in a Lossy Medium", IEEE Trans. On Antennas and prop, vol. 52, no. 8, (2004) August.

[17] E. H. Grant, "The physical interaction of electromagnetic fields with people", in Proc. Conf. Electrmag. Fields and Human Health, (1991), pp. 121-125

[18] T. J Brooks, H. Baker and K. A. Mercer. "A review of position tracking”, 1st Int Conference on Sensing Technology Palmerston North, New Zealand, (2005) November 21-23.

[19] P. Bahl and V. N. Padmanabhan, "Radar: An in-building rf-based user location and tracking system," in IEEE INFOCOM, vol. 2, (2000) March, pp. 775-784.

[20] A. Kotanen, M. Hannikainen, H. Leppakoski and T. Hamaleinen, "Positioning with IEEE 802.11b wireless lan", in 14th IEEE Proceedings on Personal, Indoor and Mobile Radio Communications (PIMRC), (2003) September, pp. 2218-2222

[21] M. Youssef, A. Agrawala and A. U. Shankar, "Wlan location determination via clustering and probability distributions", in Proceedings of the First IEEE International Conference on Pervasive Computing and Communications (PerCom), (2003) March, pp. 143-150.

[22] F. Adepoju and K. Arshak, "Capsule Position Estimation using Radio Signals", US Patent UNI026/P/IE (In press).

[23] T. H. B. V. Calculus, 2nd Ed. Prentice Hall, New Jersey; (2001), pp. 102-126. ISBN 013088005-1.

[24] W. S. Murphy and W. Hereman, "Determination of a position in three dimensions using trilateration and approximate distances", Dept of Mathematical and Computer Sciences, Colorado Schl of Mines, Golden Colorado; The Bulldozer project; MCS-91-11, (1991) Septmebr.

[25] C. L. Lawson and R. J. Hanson, "Solving Least Squares Problems", SIAM, Philadelphia, (1995), pp $107-169,190$.

[26] B. Noble and J. W. Daniel, “Applied Linear Algebra”, Englewood Cliffs: Prentice Hall, (1988).

[27] W. Navidi, W. S. Murphy and W. Hereman, "Statistical methods in Surveying by trilateration", Journal of Computational Statistics and Data Analysis, vol. 27, no. (1998), pp. 209-227.

[28] J. J. McKeown, "Specialized versus General purpose Algorithms for minimizing Functions that are sum of squared terms", Jnl of mathematical prog., vol. 9, (1975), pp. 57-68. 
[29] E. M. Mikhail, "Observations and least squares", New York: IEP-Dun-Donnelley, Harper and Row, (1976).

[30] A. Ward, A. Jones and A. Hopper, "A new location technique for the active office", in IEEE Personal Communications, vol. 4, no. 5, (1997) October, pp. 42-47.

[31] J. M Bland and D. G Altman "Measuring agreement in method comparison studies." Statistical Methods Medical Research, vol. 8, (1999), pp. 135 -160. 